\title{
A New Method to Study the Periodic Solutions of the Ordinary Differential Equations Using Functional Analysis
}

\author{
Seifedine Kadry ${ }^{1, *}$, Gennady Alferov ${ }^{2}$, , Gennady Ivanov ${ }^{2}$, Vladimir Korolev ${ }^{2}$ \\ and Ekaterina Selitskaya ${ }^{2}$ \\ 1 Department of mathematics and computer science, faculty of science, Beirut Arab University, \\ P.O. Box 11-5020 Riad El Solh, Beirut 1107 2809, Lebanon \\ 2 Faculty of Applied Mathematics and Control Processes, Sain-Petersburg State University, \\ 199034 Saint-Petersburg, Russia \\ * Correspondence: s.kadry@bau.edu.lb or skadry@gmail.com
}

Received: 5 July 2019; Accepted: 24 July 2019; Published: 29 July 2019

\begin{abstract}
In this paper, a new theorems of the derived numbers method to estimate the number of periodic solutions of first-order ordinary differential equations are formulated and proved. Approaches to estimate the number of periodic solutions of ordinary differential equations are considered. Conditions that allow us to determine both upper and lower bounds for these solutions are found. The existence and stability of periodic problems are considered.
\end{abstract}

Keywords: derived number; periodic solutions; Non-smooth analysis; Dini-Holder derivatives

\section{Introduction}

In this paper a method to study periodic solutions of first-order ordinary differential equations is developed based on the ideas of functional analysis. We eliminate the polynomial requirement of the right-hand side of the equation under study and proposed a different approach to obtain the upper and lower estimates for the number of periodic solutions. Theorems of the derived numbers method to estimate the number of periodic solutions of first-order ordinary differential equations are formulated and proved. A large number of papers have been devoted to the study of periodic solutions. The theoretical foundations of periodic solutions of differential equations were developed by A. Poincare for the three-body problem [1] and A.M. Lyapunov for the problem of any mechanical system motion [2]. Periodic solutions take an important part in the qualitative theory of differential equations and in applied problems [3]. The analysis problems for periodic solutions of differential equations arise in classical mechanics, celestial mechanics [4-15], space robotics [16-24], in modeling of economic processes [25-29]. However, there is no general approach to study periodic solutions of differential equations. There are several methods to solve this problem. Thus, the basic method to prove the existence of periodic solutions of differential equations is the Poincare-Andronov point mapping method, the method of directing functions, variational methods, the topological method, the Krylov-Bogolyubov averaging and so on. Note that these methods are difficult enough to be applied.

In this paper, the problem of estimating the number of periodic solutions of first-order differential equations is solved on the basis of the results of studies [30,31] and with the use of derived numbers theory [32,33].

\section{State of the Art}

Let the right-hand side of equation

$$
\dot{x}=f(t, x)
$$


be a continuous function with respect to the set of arguments and is function $\omega$-periodic with respect to $t$.

The first examples of this kind was N.M. Adamov. In the Report of the USSR Academy of Sciences V.A. Pliss showed that this series of theorems cannot be continued by constructing an example, on the right side of which there is a 4th order polynomial, and the equation itself has 5 periodic solutions [34]. A more general result in this direction was obtained by V.M. Lebedeva, having shown that an equation in which the right-hand side is a 4th order polynomial, can have any preassigned number of periodic solutions $[5,33]$. Our idea in this paper is to reject the requirement of polynomiality of the right-hand side of the equation under study and proposed a different approach for obtaining the upper and lower estimates for the number of periodic solutions.

\section{The Upper Bound for a Number of Periodic Solutions}

Theorem 1. If the right-hand side of Equation (1) for each fixed $t$ is a function increasing in $x$ and there exists a time point $t^{*} \in[0, \omega]$ such that $f\left(t^{*}, x\right)$ strictly increases, then Equation (1) can have no more than one periodic solution.

Proof. Suppose Equation (1) has two periodic solutions $x_{1}(t)$ and $x_{2}(t)$ contrary to the statement of the theorem. Let us show these solutions have no common points.

Since $x_{1}(t) \equiv x_{2}(t)$, then there exists a point $t_{0}$ such that $x_{1}\left(t_{0}\right) \neq x_{2}\left(t_{0}\right)$. Without loss of generality, it can be assumed that $t_{0}=0$ and

$$
x_{1}(0)<x_{2}(0) .
$$

Assume the contrary, namely, that the solutions $x_{1}$ and $x_{2}$ intersect. This means that there exists $T>0$, such that $x_{1}(T)=x_{2}(T)$. Let $t^{\prime}$ denote an exact lower bound of set $\left\{t: x_{1}(t)=x_{2}(t), t>0\right\}$. The continuity of functions $x_{1}(t)$ and $x_{2}(t)$ and condition (2) implies that $t^{\prime}>0$.

From inequality (2) and the point $t^{\prime}$ selection it follows that for $t \in\left[0, t^{\prime}\right] . x_{1}(t) \leq x_{2}(t)$. By the hypothesis of the theorem, the function $f(t, x)$ increases with respect $x$ for each fixed $t$, and, consequently, for $t \in\left[0, t^{\prime}\right]$ the following the inequality is realized

$$
f\left(t, x_{1}(t)\right) \leq f\left(t, x_{2}(t)\right) .
$$

The subtraction $x_{2}\left(t^{\prime}\right)-x_{1}\left(t^{\prime}\right)$ can be represented as:

$$
x_{2}\left(t^{\prime}\right)-x_{1}\left(t^{\prime}\right)=x_{2}(0)-x_{1}(0)+\int_{0}^{t^{\prime}}\left[f\left(t, x_{2}(t)\right)-f\left(t, x_{1}(t)\right)\right] d t .
$$

From (3) it follows that

$$
\int_{0}^{t^{\prime}}\left[f\left(t, x_{2}(t)\right)-f\left(t, x_{1}(t)\right)\right] d t \geq 0
$$

but due to (2) the following is obtained

$$
x_{1}\left(t^{\prime}\right)<x_{2}\left(t^{\prime}\right),
$$

which contradicts the selection of the point $t^{\prime}$.

Thus, for $t \geq 0$ the solutions $x_{1}$ and $x_{2}$ do not intersect. These solutions do not have common points for $t<0$, which follows from their $\omega$-periodicity.

So, if Equation (1) has two $\omega$-periodic solutions and inequality (2) is realised, then for all $t$

$$
x_{1}(t)<x_{2}(t),
$$


and, consequently, inequality (3) is realized for all $t$. And then

$$
\int_{0}^{\omega}\left[f\left(t, x_{2}(t)\right)-f\left(t, x_{1}(t)\right)\right] d t \geq 0
$$

Moreover, since $f(t, x)$ is continuous and $f\left(t^{*}, x\right)$ is strictly increasing in $x$, in some neighborhood of the point $t^{*} \in[0, \omega]$ a strict inequality is realised:

$$
f\left(t, x_{1}(t)\right)<f\left(t, x_{2}(t)\right)
$$

therefore, we can assert that

$$
\int_{0}^{\omega}\left[f\left(t, x_{2}(t)\right)-f\left(t, x_{1}(t)\right)\right] d t>0
$$

Taking into account the last inequality, the following contradictory chain of relations is obtained:

$$
0=\left[x_{2}(\omega)-x_{2}(0)\right]-\left[x_{1}(\omega)-x_{1}(0)\right]=\int_{0}^{\omega}\left[f\left(t, x_{2}(t)\right)-f\left(t, x_{1}(t)\right)\right] d t>0
$$

This contradiction approves the assumption that Equation (1) can have two different $\omega$-periodic solutions.

Example 1. After approving a single periodic solution for Equation (1), consider the case:

$$
\dot{x}=f(x)=x-1 \text {. }
$$

The first derivative of the right side of this equation is positive $\frac{d f}{d x}=1$ and therefore, this equation can have at most one periodic solution. In our case this solution: $x=1$.

Theorem 2. If the right-hand side of (1) is a convex function for each fixed $t$, and $t^{*} \in[0, \omega]$ is strictly convex with respect to $x$ for some $t^{*} \in[0, \omega]$, then Equation (1) can have no more than two different $\omega$-periodic solutions.

Proof. First of all, let us proove that the Equation (1) has the property of existence and uniqueness for the solution under the conditions of the theorem. Assume for that purpose that if $f$ is convex and continuous, then it is a Lipschitz function.

Indeed, let $\left(t_{0}, x_{0}\right)$ be an arbitrary point of the plane, and let $\tau$ and $\delta$ be some positive numbers. Let us show that for the domain $D=\left\{(t, x): t \in\left[t_{0}-\tau, t_{0}+\tau\right], x \in\left[x_{0}-\delta, x_{0}+\delta\right]\right\}$ there exists $L$, such that for all $x^{\prime}, x^{\prime \prime} \in\left[x_{0}-\delta, x_{0}+\delta\right]$ and for all $t \in\left[t_{0}-\tau, t_{0}+\tau\right]$

$$
\left|f\left(t, x^{\prime \prime}\right)-f\left(t, x^{\prime}\right)\right| \leq L\left|x^{\prime \prime}-x^{\prime}\right| \text {. }
$$

In the proof of Theorem 14 [31] it is noted that for each fixed $t$ function $f$ has a bounded right derivative, which is an increasing function with respect to $x$. Let us show that there exists $K$, such that for all $(t, x) \in D$

$$
\left|f^{\prime+}(t, x)\right| \leq K
$$

Since for each fixed $t$ function $f^{\prime+}(t, x)$ increases monotonically, it can take its extreme values only at the ends of the interval. Therefore, in order to prove the required inequality, it suffices to show that the functions $f^{\prime+}\left(t, x_{0}-\delta\right)$ and $f^{\prime+}\left(t, x_{0}+\delta\right)$ as $t \in\left[t_{0}-\tau, t_{0}+\tau\right]$ are bounded. 
Let us prove that $f^{\prime+}\left(t, x_{0}+\delta\right)$ is bounded from above. Consider some $m>x_{0}+\delta$. Then, taking into account the fact that for any fixed $t$ function $\frac{f(t, y)-f\left(t, x_{0}+\delta\right)}{y-x_{0}-\delta}$ increases with respect to $y$, for all $y \in\left(x_{0}+\delta, m\right]$

$$
\begin{gathered}
\frac{f(t, y)-f\left(t, x_{0}+\delta\right)}{y-x_{0}-\delta} \leq \frac{f(t, m)-f\left(t, x_{0}+\delta\right)}{m-x_{0}-\delta} \\
\leq \frac{|f(t, m)|+\left|f\left(t, x_{0}+\delta\right)\right|}{m-x_{0}-\delta} .
\end{gathered}
$$

The function $f$ is continuous, and consequently, $|f(t, m)|$ and $\left|f\left(t, x_{0}+\delta\right)\right|$ are bounded on $\left[t_{0}-\tau, t_{0}+\tau\right]$. Thus, it can be concluded that there exists some constant $K$, such that for all $y \in\left(x_{0}+\delta, m\right]$ and all $t \in\left[t_{0}-\tau, t_{0}+\tau\right]$

$$
\frac{f(t, y)-f\left(t, x_{0}+\delta\right)}{y-x_{0}-\delta} \leq K
$$

But then for all $t \in\left[t_{0}-\tau, t_{0}+\tau\right]$

$$
f^{\prime+}\left(t, x_{0}+\delta\right) \leq K
$$

The fact that $f^{\prime+}\left(t, x_{0}-\delta\right)$ is bounded from below can be proved in a similar way.

Combining these two results and taking into account that for each $t$ function $f^{\prime+}$ is increasing with respect to $x$, the statement on the boundedness of the function $f^{\prime+}$ on $D$ is valid becomes clear.

Consider two arbitrary points $x^{\prime}$ and $x^{\prime \prime}$ in $\left[x_{0}-\delta, x_{0}+\delta\right]$ and an arbitrary moment $t^{\prime}$ in $\left[t_{0}-\tau, t_{0}+\tau\right]$. The function $f\left(t^{\prime}, x\right)$ is continuous on $\left[x^{\prime}, x^{\prime \prime}\right]$ and has a finite right derivative. But due to Theorem 12 [33] it implies existence of points $x_{1}, x_{2} \in\left(x^{\prime}, x^{\prime \prime}\right)$, such that

$$
f^{\prime+}\left(t^{\prime}, x_{1}\right) \leq \frac{f\left(t^{\prime}, x^{\prime \prime}\right)-f\left(t^{\prime}, x^{\prime}\right)}{x^{\prime \prime}-x^{\prime}} \leq f^{\prime+}\left(t^{\prime}, x_{2}\right) .
$$

Now, taking into account the boundedness of the function $f^{\prime+}$ on $D$ and arbitrariness of $x^{\prime}, x^{\prime \prime}$ and $t^{\prime}$, it follows that for any $L \geq K$ and arbitrary $x^{\prime}, x^{\prime \prime} \in\left[x_{0}-\delta, x_{0}+\delta\right]$

$$
\begin{gathered}
\left|f\left(t, x^{\prime \prime}\right)-f\left(t, x^{\prime}\right)\right| \leq L\left|x^{\prime \prime}-x^{\prime}\right|, \\
t \in\left[t_{0}-\tau, t_{0}+\tau\right],
\end{gathered}
$$

which means that $f$ is a Lipschitzian function.

Let the conditions of the theorem be satisfied. Let us show that in this case the Equation (1) can not have more than two $\omega$-periodic solutions. Assume, by contradiction, that the Equation (1) has three $\omega$-periodic solutions $y_{1}, y_{2}$ and $y_{3}$. As shown above, the function $f$ satisfies the Lipschitz condition, and consequently, the Equation (1) has the property of existence and uniqueness for solutions, and therefore, if for $t=0$

$$
y_{1}(0)<y_{2}(0)<y_{3}(0)
$$

then for any $t$

$$
y_{1}(t)<y_{2}(t)<y_{3}(t) .
$$

Consider two obvious identities, which in view of (4) are valid for all $t$ :

$$
\frac{\dot{y}_{3}-\dot{y}_{2}}{y_{3}-y_{2}}=\frac{f\left(t, y_{3}\right)-f\left(t, y_{2}\right)}{y_{3}-y_{2}}
$$

and

$$
\frac{\dot{y}_{2}-\dot{y}_{1}}{y_{2}-y_{1}}=\frac{f\left(t, y_{2}\right)-f\left(t, y_{1}\right)}{y_{2}-y_{1}} .
$$


Subtracting the second equality from the first one, the following is obtained:

$$
\frac{\dot{y}_{3}-\dot{y}_{2}}{y_{3}-y_{2}}-\frac{\dot{y}_{2}-\dot{y}_{1}}{y_{2}-y_{1}}=\frac{f\left(t, y_{3}\right)-f\left(t, y_{2}\right)}{y_{3}-y_{2}}-\frac{f\left(t, y_{2}\right)-f\left(t, y_{1}\right)}{y_{2}-y_{1}} \text {. }
$$

Let us represent the solution of Equation (1) $y_{2}$ in the form:

$$
y_{2}(t)=\alpha(t) y_{1}(t)+(1-\alpha(t)) y_{3}(t)
$$

By (4) it is obvious that $\alpha(t) \in(0,1)$ for all $t$.

Substituting the representation (6) into the right-hand side of (5), the following is obtained:

$$
\begin{gathered}
\frac{f\left(t, y_{3}\right)-f\left(t, y_{2}\right)}{y_{3}--y_{2}}-\frac{f\left(t, y_{2}\right)-f\left(t, y_{1}\right)}{y_{2}-y_{1}}= \\
\frac{f\left(t, y_{3}\right)-f\left(t, \alpha y_{1}+(1-\alpha) y_{3}\right)}{\alpha\left(y_{3}-y_{1}\right)} \\
-\frac{f\left(t, \alpha y_{1}+(1-\alpha) y_{3}\right)-f\left(t, y_{1}\right)}{(1-\alpha)\left(y_{3}-y_{1}\right)} \\
=\frac{\alpha f\left(t, y_{1}\right)+(1-\alpha) f\left(t, y_{3}\right)-f\left(t, \alpha y_{1}+(1-\alpha) y_{3}\right)}{\alpha(1-\alpha)\left(y_{3}-y_{1}\right)} \\
=g(t) .
\end{gathered}
$$

The function $g(t)$ is continuous, since the functions $f$ and $\alpha$ are continuous and since it follows from inequality (4) and the definition of $\alpha$ that there exists a number $\beta>0$, such that for all $t$

$$
\alpha(t) 1(-\alpha(t))\left(y_{3}(t)-y_{1}(t)\right) \geq \beta .
$$

Moreover, taking into account the convexity of the function $f$, i.e., that for all $t$

$$
\alpha f\left(t, y_{1}\right)+(1-\alpha) f\left(t, y_{3}\right) \geq f\left(t, \alpha y_{1}+(1-\alpha) y_{3}\right),
$$

it can be concluded that for all $t$

$$
g(t) \geq 0
$$

But, by the assertion of the theorem, $f\left(t^{*}, x\right)$ is strictly convex for $t=t^{*} \in[0, \omega]$, which implies that as at the point $t^{*}$ itself, as in some neighborhood of it, by continuity the following strict inequality is realized:

$$
g(t)>0 \text {. }
$$

Thus, the function $g$ is continuous, non-negative and there is an interval which the function $g$ takes only positive values in.

Considering this, let the identity (5) be integrated in the range from 0 to $\omega$. Since the functions $y_{1}$, $y_{2}$ and $y_{3}$ are $\omega$-periodic, the following is obtained:

$$
0<\int_{0}^{\omega} g(t) d t=\int_{0}^{\omega} \frac{\dot{y}_{3}-\dot{y}_{2}}{y_{3}-y_{2}}-\frac{\dot{y}_{2}-\dot{y}_{1}}{y_{2}-y_{1}} d t=\ln \left(\frac{y_{3}(\omega)-y_{2}(\omega)}{y_{3}(0)-y_{2}(0)}\right)-\ln \left(\frac{y_{2}(\omega)-y_{1}(\omega)}{y_{2}(0)-y_{1}(0)}\right)=0 .
$$

This contradiction shows that if the conditions of the theorem are satisfied, the Equation (1) cannot have three $\omega$-periodic solutions.

Example 2. For the following statement, consider the case: $\dot{x}=x^{2}-3 x+2$.

In this example, the second derivative is positive, and therefore this equation can have no more than two periodic solutions. These decisions: $x=1$ and $x=2$. 
Theorem 3. If the right-hand side of the Equation (1) has a derivative $\frac{d}{d x} f(t, x)=f^{\prime}(t, x)$ continuous in the set of arguments, which is a function convex with respect to $x$ for each $t$ and there exists a moment $t^{*} \in[0, \omega]$ such that $f^{\prime}\left(t^{*}, x\right)$ is strictly convex, then the Equation (1) cannot have more than three $\omega$-periodic solutions.

Proof. Let $\phi(t, x)$ denote a solution of the Equation (1), that starts at the point $x$ at $t=0$. Then $\phi(\omega, x)$ is a point which this solution at $t=\omega$ gets in. Let an auxiliary $\psi(x)$ function be introduced, considering that $\psi(x)=\ln \phi^{\prime}(\omega, x)$, where prime mark means $\frac{d}{d x}$.

Since the function $f^{\prime}$ is defined and continuous, then the function $\phi^{\prime}$ is also defined and continuous, and from the equation in variations it follows that for any $x$ and $t$

$$
\phi^{\prime}(t, x)>0 \text {. }
$$

Thus, the function $\psi(x)$ is specified correctly.

If it is additionally known that $\psi(x)$ is strictly convex, then the theorem is very simple to prove. Indeed, let the Equation (1) have four $\omega$-periodic solutions $\phi\left(t, x_{i}\right)$ beginning at points $x_{i}$, $i=1,2,3$, 4. Let, for definiteness,

$$
x_{1}<x_{2}<x_{3}<x_{4} .
$$

Let a function $\phi(\omega, x)-x$ be constructed. It is continuous at each interval $\left[x_{j}, x_{j+1}\right] j=1,2,3$, takes the same values at the ends of the interval, since $\phi\left(\omega, x_{i}\right)=x_{i}, i=1,2,3,4$ due to $\omega$-periodicity, and finally, it has a derivative at each point $x$. Thus, for the function

$$
g(x)=\phi(\omega, x)-x
$$

all the conditions of Rolle's theorem are satisfied, and, consequently, there exist points

$$
z_{1} \in\left(x_{1}, x_{2}\right), \quad z_{2} \in\left(x_{2}, x_{3}\right), \quad z_{3} \in\left(x_{3}, x_{4}\right),
$$

such that

$$
g^{\prime}\left(z_{j}\right)=0, \quad j=1,2,3
$$

This implies that

$$
\phi^{\prime}\left(\omega, z_{j}\right)=1, \quad j=1,2,3
$$

from which it is finally obtained that

$$
\psi\left(z_{j}\right)=0, \quad j=1,2,3 .
$$

So, if the Equation (1) has four $\omega$-periodic solutions, then the function $\psi(x)$ takes the same values at three different points, which contradicts the assumption of its convexity. Therefore, in order to prove the theorem, it is sufficient to show that the function $\psi(x)$ is strictly convex on $\left[x_{1}, x_{4}\right]$.

From the equation in variations it follows that

$$
\psi(x)=\int_{0}^{\omega} f^{\prime}(t, \phi(t, x)) d t .
$$

By stated conditions, the function $f^{\prime}(t, x)$ is convex with respect to $x$ and, therefore, has a finite right derivative at any point $x$ at each fixed $t$ due to by Theorem 14 [33]. The function $\phi(t, x)$, by virtue of the existence and uniqueness of the solutions of Equation (1), strictly increases with respect to each fixed. It follows that the right derivative of the function, which we denote by, can be written in the form. 
The function $\phi(t, x)$ strictly increases with respect to $x$ at each fixed $t$ by virtue of the existence and uniqueness of solutions of the Equation (1). This implies that the right derivative of the function $f^{\prime}(t, \phi(t, x))$ denoted here by $\left(f^{\prime}(t, \phi(t, x))\right)^{\prime+}$ can be represents in the following form

$$
\left(f^{\prime}(t, \phi(t, x))\right)^{\prime+}=\left(f^{\prime}\right)^{\prime+}(t, \phi(t, x)) \phi^{\prime}(t, x),
$$

where $\left(f^{\prime}\right)^{\prime+}(t, \phi(t, x))$ denotes the right derivative of the function $f^{\prime}(t, \phi)$ with respect to the variable $\phi$ at the point $\phi=\phi(t, x)$. Then

$$
\psi^{\prime+}(x)=\int_{0}^{\omega}\left(f^{\prime}\right)^{\prime+}(t, \phi(t, x)) \phi^{\prime}(t, x) d t
$$

On the set

$$
D=\left\{(t, x): t \in[0, \omega], x \in\left[x_{1}, x_{4}\right]\right\}
$$

the function $\phi(t, x)$ is bounded, since it increases with respect to $x$ by virtue of the existence and uniqueness of the solutions of Equation (1), and, consequently,

$$
\phi\left(t, x_{1}\right) \leq \phi(t, x) \leq \phi\left(t, x_{4}\right),
$$

where $\phi\left(t, x_{1}\right)$ and $\phi\left(t, x_{4}\right)$ are bounded on $[0, \omega]$ as the continuous $\omega$-periodic solutions of the Equation (1). Then the function $f^{\prime}(t, \phi(t, x))$ is also bounded on $D$ as a function continuous in the set of arguments, and this entails the boundedness of the function

$$
\phi^{\prime}(t, x)=e^{\int_{0}^{t} f^{\prime}(\tau, \phi(\tau, x)) d \tau}
$$

on $D$.

Further, the proof of Theorem 2 implies that the function $\left(f^{\prime}\right)^{\prime+}(t, \phi(t, x))$ is bounded on $D$, since it is a right derivative of a function convex in $x$ and continuous in the set of arguments. Thus, all the functions entering the right-hand side of (7) are bounded, and therefore, the function $\psi^{\prime+}$ is bounded on $\left[x_{1}, x_{4}\right]$.

Thus, $\psi$ is a function continuous on $\left[x_{1}, x_{4}\right]$ that has a bounded right derivative on this interval. Then, according to Theorem 14 [33], in order to prove the strict convexity of the function $\psi$ on $\left[x_{1}, x_{4}\right]$, it is sufficient to show that $\psi^{\prime+}$ strictly increases on $\left[x_{1}, x_{4}\right]$.

Let $\lambda^{+}\left[\psi^{+}\right](x)$ be an arbitrary right-hand derived number of the function $\psi^{\prime+}$ at the point $x \in\left[x_{1}, x_{4}\right],\left\{h_{n}\right\}$ be a sequence which this number is realized on, and $h_{n} \rightarrow 0$ as $n \rightarrow \infty, h_{n}>0$. Let us prove that

$$
\lambda^{+}\left[\psi^{\prime+}\right](x) \geq 0
$$

Indeed, considering that function $\left(f^{\prime}\right)^{\prime+}(t, \phi(t, x))$ increases with respect to $x, \phi^{\prime}(t, x)>0$ and using the equation in variations, the following is obtained:

$$
\begin{aligned}
& \lambda^{+}\left[\psi^{\prime+}\right](x)=\lim _{n \rightarrow \infty} \frac{1}{h_{n}}\left(\int_{0}^{\omega}\left(f^{\prime}\right)^{\prime+}\left(t, \phi\left(t, x+h_{n}\right)\right) \phi^{\prime}\left(t, x+h_{n}\right)-\left(f^{\prime}\right)^{\prime+}(t, \phi(t, x)) \phi^{\prime}(t, x) d t\right) \\
& =\lim _{n \rightarrow \infty} \frac{1}{h_{n}} \int_{0}^{\omega} \phi^{\prime}\left(t, x+h_{n}\right)\left[\left(f^{\prime}\right)^{\prime+}\left(t, \phi\left(t, x+h_{n}\right)\right)\right. \\
& \left.-\left(f^{\prime}\right)^{\prime+}(t, \phi(t, x))\right] d t+\lim _{n \rightarrow \infty} \int_{0}^{\omega}\left(f^{\prime}\right)^{\prime+}(t, \phi(t, x)) \\
& \frac{\phi^{\prime}\left(t, x+h_{n}\right)-\phi^{\prime}(t, x)}{h_{n}} d t \geq \\
& \int_{0}^{\omega}\left(f^{\prime}\right)^{\prime+}(t, ?(t, x))\left(\varphi^{\prime}\right)^{\prime+}(t, x) d t=\int_{0}^{\omega}\left[\left(f^{\prime}\right)^{\prime+}(t, \phi(t, x)) \phi^{\prime}(t, x)\right] \\
& \frac{\left(\phi^{\prime}\right)^{\prime+}(t, x)}{\phi^{\prime}(t, x)} d t=\int_{0}^{\omega} \frac{d}{d t}\left[\psi^{\prime+}(t, x)\right] \psi^{\prime+}(t, x) d t \\
& =\frac{1}{2} \psi^{\prime+2}(x) \geq 0 .
\end{aligned}
$$


Suppose here that

$$
\psi^{\prime+}(t, x)=\frac{\left(\phi^{\prime}\right)^{\prime+}(t, x)}{\phi^{\prime}(t, x)}=\int_{0}^{t}\left(f^{\prime}\right)^{\prime+}(\tau, \phi(\tau, x)) \phi^{\prime}(\tau, x) d \tau
$$

and consider that

$$
\psi^{+}(\omega, x)=\psi^{\prime+}(x)
$$

The limiting transition under the integral sign is admissible, since the right derivative $\left(\phi^{\prime}\right)^{\prime+}$ of the function $\phi^{\prime}$ exists and it is finite on $\left[x_{1}, x_{4}\right]$.

Repeating these arguments, for an arbitrary left derived number of the function $\psi^{\prime}$ at a point $x$, the following estimation is obtained:

$$
\lambda^{-}\left[\psi^{\prime+}\right](x) \geq \int_{0}^{\omega}\left(f^{\prime}\right)^{\prime+}(t, \phi(t, x))\left(\phi^{\prime}\right)^{\prime-}(t, x) d t .
$$

Since the function $\left(\phi^{\prime}\right)^{-}$is bounded on

$$
D=\left\{(t, x): t \in[0, \omega], x \in\left[x_{1}, x_{4}\right]\right\},
$$

then the function $\lambda^{-}\left[\psi^{+}\right]$is bounded below on $\left[x_{1}, x_{4}\right]$.

Thus, all the right derivatives of the function $\left[x_{1}, x_{4}\right]$ are nonnegative on $\left[x_{1}, x_{4}\right]$, and all its left derived numbers are uniformly bounded below. Therefore, according to Theorem 9 [32], the function $\psi^{+}$increases on $\left[x_{1}, x_{4}\right]$.

Let us show that $\psi^{\prime+}$ strictly increases on $\left[x_{1}, x_{4}\right]$. First of all, note that from the estimation obtained above

$$
\lambda^{+}\left[\psi^{\prime+}\right](x) \geq \frac{1}{2} \psi^{\prime+2}(x)
$$

it follows that if the point $x_{0}$ is not a root of the function $\psi^{+}$, then

$$
\lambda^{+}\left[\psi^{\prime+}\right]\left(x_{0}\right)>0,
$$

and if there are no roots of the function $\psi^{+}$in a neighborhood of the point $x_{0}$, then the function $\psi^{\prime+}$ strictly increases in this neighborhood.

Suppose that $\psi^{\prime+}$ increases, but not strictly. Then there exists a segment $\left[y_{1}, y_{2}\right]$ which the function $\psi^{\prime+}$ is constant on. But, as follows from the remark made above, this segment can be only zero, since if there is a neighborhood with $\psi^{\prime+} \neq 0$, then $\psi^{\prime+}$ increases strictly in it. Then,

$$
\psi^{\prime+}(x) \equiv 0, \quad x \in\left[y_{1}, y_{2}\right] .
$$

Then for the right derivative of the function $\psi^{\prime+}$ at an arbitrary point $x \in\left[y_{1}, y_{2}\right]$

$$
\begin{aligned}
& 0=\left(\psi^{++}\right)^{\prime+}(x)= \\
& \lim _{h \rightarrow+0} h^{-1} \int_{0}^{\omega}\left(f^{\prime}\right)^{\prime+}(t, \phi(t, x+h)) \phi^{\prime}(t, x+h)- \\
& -\left(f^{\prime}\right)^{\prime+}(t, \phi(t, x)) \phi^{\prime}(t, x) d t=\lim _{h \rightarrow+0} \frac{1}{h} \int_{0}^{\omega} \phi^{\prime}(t, x+h)\left[\left(f^{\prime}\right)^{\prime+}\right. \\
& \left.(t, \phi(t, x+h))-\left(f^{\prime}\right)^{\prime+}(t, \phi(t, x))\right] d t+ \\
& \int_{0}^{\omega}\left(f^{\prime}\right)^{\prime+}(t, \phi(t, x))\left[\lim _{h \rightarrow+0} \frac{\phi^{\prime}(t, x+h)-\phi^{\prime}(t, x)}{h}\right] d t .
\end{aligned}
$$

As mentioned above, the second term is $\frac{1}{2} \psi^{\prime+2}(x)=0$, therefore, the first term denoted by $\mathrm{I}(\mathrm{x})$ is also equal to zero. 
Estimating $I(x)$ from below, the following is realized:

$$
0=I(x) \geq \lim _{h \rightarrow+0} h^{-1} \int_{0}^{\omega} m\left[\left(f^{\prime}\right)^{\prime+}(t, \phi(t, x+h))-\left(f^{\prime}\right)^{\prime+}(t, \phi(t, x))\right] d t \geq 0,
$$

where

$$
m=\min _{t \in[0, \omega], x \in\left[x_{1}, x_{4}\right], h \in[0, \delta]} \phi^{\prime}(t, x+h) .
$$

Let us show that $m>0$.

By virtue of the theorem on integral continuity for an arbitrarily chosen $\varepsilon>0$ consider $\delta>0$, such that for $\left|x_{1}-x\right| \leq \delta$

$$
\left|\phi\left(t, x_{1}\right)-\phi(t, x)\right|<\varepsilon
$$

for all $t \in[0, \omega]$. It can assumed that the chosen $\varepsilon$ and $\delta$ are also suitable for the solution $\phi\left(t, x_{4}\right)$. The solutions of the Equation (1) $\phi\left(t, x_{1}\right)$ and $\phi\left(t, x_{4}\right)$ are bounded on $[0, \omega]$ as periodic. Considering that $\phi(t, x)$ increases with respect to $x$ for each fixed $t \in[0, \omega]$, it follows that $\phi(t, x)$ is bounded on $d$. Therefore, for $\delta$ considered above $\phi(t, x)$ is limited on

$$
D^{\prime}=\left\{(t, x): t \in[0, \omega], x \in\left[x_{1}, x_{4}+\delta\right]\right\} .
$$

Further, the function $f^{\prime}$ is continuous in the set of arguments by condition and, therefore, it is also bounded on $D^{\prime}$. But then there exists $A$, such that for any $t \in[0, \omega], x \in\left[x_{1}, x_{4}\right]$ and $h \in[0, \delta]$

$$
\left|\int_{0}^{t} f^{\prime}(\tau, \phi(\tau, x+h)) d \tau\right| \leq A
$$

Considering that

$$
\phi(t, x)=e^{\int_{0}^{t} f^{\prime}(\tau, \phi(\tau, x)) d \tau},
$$

it follows that

$$
m \geq e^{-A}>0 .
$$

The positiveness of $m$ and fairness of equality

$$
\lim _{h \rightarrow+0} h^{-1} \int_{0}^{\omega} m\left[\left(f^{\prime}\right)^{\prime+}(t, \phi(t, x+h))-\left(f^{\prime}\right)^{\prime+}(t, \phi(t, x))\right] d t=0
$$

imply that

$$
\lim _{h \rightarrow+0} h^{-1} \int_{0}^{\omega}\left[\left(f^{\prime}\right)^{\prime+}(t, \phi(t, x+h))-\left(f^{\prime}\right)^{\prime+}(t, \phi(t, x))\right] d t=0 .
$$

Consider a function $r(x)$ setting that

$$
r(x)=\int_{0}^{\omega}\left(f^{\prime}\right)^{\prime+}(t, \phi(t, x)) d t .
$$

From the equality obtained above it follows that

$$
r^{\prime}=0 .
$$

It is easy to see that the left derivatives of the function $r$ are nonnegative. Besides, repeating for the left derivative of the function $\psi^{\prime+}$ the arguments similar with ones for the right derivative of this function, it is obvious that the left derivative of the function is also bounded above. Thus, on the basis of Theorem 1 we conclude that the function $r$ is continuous on $\left[y_{1}, y_{2}\right]$, and then it follows that $r$ is constant on $\left[y_{1}, y_{2}\right]$. 
Let us proove that

$$
r\left(y_{1}\right)<r\left(y_{2}\right)
$$

Consider the difference

$$
r\left(y_{2}\right)-r\left(y_{1}\right)=\int_{0}^{\omega}\left[\left(f^{\prime}\right)^{\prime+}\left(t, \phi\left(t, y_{2}\right)\right)-\left(f^{\prime}\right)^{\prime+}\left(t, \phi\left(t, y_{1}\right)\right)\right] d t .
$$

$\phi\left(t, y_{2}\right)>\phi\left(t, y_{1}\right)$ due to uniqueness. Hence,

$$
\left(f^{\prime}\right)^{\prime+}\left(t, \phi\left(t, y_{2}\right)\right)-\left(f^{\prime}\right)^{\prime+}\left(t, \phi\left(t, y_{1}\right)\right) \geq 0,
$$

since the function $f^{\prime}$ is convex by condition, then $\left(f^{\prime}\right)^{\prime+}$ increases by Theorem 14 [33]. Moreover, by the condition of the theorem there exists a point $t^{*}$ at which the function $f^{\prime}$ is strictly convex, and therefore at this point

$$
\left(f^{\prime}\right)^{\prime+}\left(t^{*}, \phi\left(t^{*}, y_{2}\right)\right)-\left(f^{\prime}\right)^{\prime+}\left(t^{*}, \phi\left(t^{*}, y_{1}\right)\right)>0 .
$$

This inequality must also hold in some neighborhood of the point $t^{*}$.

Indeed, if this is not so, then there exists a sequence $\left\{t_{k}\right\}$ converging to $t^{*}$, such that for any $k$

$$
\left(f^{\prime}\right)^{\prime+}\left(t_{k}, \phi\left(t_{k}, y_{1}\right)\right)=\left(f^{\prime}\right)^{\prime+}\left(t_{k}, \phi\left(t_{k}, y_{2}\right)\right) .
$$

But then, for any $\alpha \in(0,1)$ and for all $k$ the following equality is realized:

$$
f^{\prime}\left(t_{k}, \alpha \phi\left(t_{k}, y_{1}\right)+(1-\alpha) \phi\left(t_{k}, y_{2}\right)\right)=\alpha f^{\prime}\left(t_{k}, \phi\left(t_{k}, y_{1}\right)\right)+(1-\alpha) f^{\prime}\left(t_{k}, \phi\left(t_{k}, y_{2}\right)\right) .
$$

Hence, in view of the continuity of the functions $f^{\prime}$ and $\phi$ in the set of arguments, it follows that

$$
f^{\prime}\left(t^{*}, \alpha \phi\left(t^{*}, y_{1}\right)+(1-\alpha) \phi\left(t^{*}, y_{2}\right)\right)=\alpha f^{\prime}\left(t^{*}, \phi\left(t^{*}, y_{1}\right)\right)+(1-\alpha) f^{\prime}\left(t^{*}, \phi\left(t^{*}, y_{2}\right)\right),
$$

but this contradicts the strict convexity of the function $f^{\prime}$ at the point $t^{*}$.

Considering the arguments given above, it follows that

$$
r\left(y_{1}\right)<r\left(y_{2}\right) .
$$

This contradiction shows that there is no interval in which $\psi^{\prime+}$ is constant, and consequently, $\psi^{\prime+}$ strictly increases in $\left[x_{1}, x_{4}\right]$. As noted above, strict increase of the function $\psi^{\prime+}$ implies a strict convexity of the function $\psi$ on $\left[x_{1}, x_{4}\right]$, which is incompatible with the assumption of the theorem that there exist four $\omega$-periodic solutions of Equation (1). of the proof of the theorem

Let $f(t, x)$ be a function continuous in the set of arguments and $\omega$-periodic with respect to t. Consider

$$
\begin{gathered}
f^{-1}(t, x)=\int_{0}^{x} f(t, y) d y, \\
f^{0}(t, x)=f(t, x), \\
f^{1}(t, x)=f^{\prime}(t, x) .
\end{gathered}
$$

Then all the obtained results can be represented in a unified form.

Example 3. For the following statement, consider the case: $\dot{x}=x^{3}-6 x^{2}+11 x-6$.

In this example, the third derivative is positive, and therefore this equation can have no more than three periodic solutions. These solutions to his statements consider the case: $x=1, x=2$ and $x=3$.

Theorem 4. If for some $k=1,2,3$ function $f^{k-2}(t, x)$ is continuous in the set of arguments and convex in $x$ for each fixed $t$, and there exists a time point $t^{*}$, such that $f^{k-2}\left(t^{*}, x\right)$ is strictly convex, then Equation (1) can have no more than $k \omega$-periodic solutions. 


\section{Lower Bound of $\omega$-Periodic Solutions Number}

In [3] V.A. Pliss constructed an example demonstrating that this series of theorems cannot be continued. Here we present another approach, which let obtain the information on a number of $\omega$-periodic solutions of the Equation (1).

Theorem 5. Let the right-hand side of the Equation (1) be such that equation

$$
f(t, \gamma(t)) \equiv 0
$$

has $n$ solutions $\gamma_{i}(t), i=1,2, \ldots, n$ with the following property:

For any, $k=0,1,2, \ldots, n$ in the domain

$$
D_{k}=\left\{(t, x): t \in(-\infty,+\infty), \gamma_{k}(t)<x<\gamma_{k+1}(t)\right\}
$$

function $f(t, x)$ is of constant sign, and the sign of the function changes when transferring to the neighboring domain. Then, if

$$
\begin{gathered}
\beta_{j}=\max _{t} \gamma_{j}(t)<\min _{t} \gamma_{j+1}(t)=\alpha_{j+1}, \\
j=1,2, \ldots, n-1,
\end{gathered}
$$

then the Equation (1) has no less than $n \omega$-periodic solutions. Here it is assumed that $\gamma_{0}=-\infty$, $\gamma_{n+1}=+\infty$.

Proof. Consider an arbitrary $i=1,2, \ldots, n$ and, for definiteness, assume that $f(t, x) \leq 0$ in the domain $D_{i-1} f(t, x) \leq 0$ and $f(t, x) \geq 0$ in $D_{i} f(t, x) \geq 0$.

By the theorem condition

$$
\beta_{j}<\alpha_{j+1}, \quad j=1,2, \ldots, n-1 .
$$

Therefore, for selected $i$ in $x=\alpha_{i}$

$$
G(x)(t)=\dot{x}-f(t, x) \geq 0,
$$

and in $x=\beta_{i}$

$$
G(x)(t) \leq 0 .
$$

But then, as shown by H. W. Knobloch в[30] иј. Mawhin в[31], in the interval

$$
\alpha_{i} \leq x \leq \beta_{i}
$$

there exists at least one $\omega$-periodic solution of the Equation (1). Since there are $n$ such intervals, then Equation (1) has no less than $n \omega$-periodic solutions.

Combining Theorem 5 with the statements in [32,33], a number of statements can be proved giving more exact information on the number of $\omega$-periodic solutions of the Equation (1). As and example, let us prove one such statement.

Example 4. Choose $n$ of arbitrary functions $\gamma_{i}(t)$ satisfying all the requirements of Theorem 5 . Then the equation

$$
\dot{x}=\prod_{i=1}^{n}\left(x-\gamma_{i}(t)\right)
$$


will have at least $n$ periodic solutions.

Theorem 6. Let the right-hand side of the Equation (1) satisfy all the requirements of Theorem 5, and for any $i=1,2, \ldots, n f(t, x)$ is a function increasing or decreasing in $x$ in the interval

$$
\alpha_{i} \leq x \leq \beta_{i}
$$

Then the Equation (1) has $n \omega$-periodic solutions.

Proof. Consider an arbitrary $i=1,2, \ldots, n$ and, for definiteness, assume that in the interval $\square$

$$
\alpha_{i} \leq x \leq \beta_{i}
$$

$f(t, x)$ increases in $x$ for each fixed $t$. Let us show that in the selected interval there is only one $\omega$-periodic solution of the Equation (1).

Let, on the contrary, in the interval

$$
\alpha_{i} \leq x \leq \beta_{i}
$$

there are two $\omega$-periodic solutions of the Equation (1) $y_{1}(t)$ and $y_{2}(t)$. As shown in the proof of Theorem 1, these solutions do not intersect anywhere. Therefore, without loss of generality, it can be assumed that for all

$$
y_{1}(t)<y_{2}(t)
$$

which implies the fulfillment of inequality

$$
f\left(t, y_{1}(t)\right) \leq f\left(t, y_{2}(t)\right), \quad t \in[0, \omega] .
$$

by the assumption of the monotonicity of the function $f(t, x)$

Taking this fact into account, it can be concluded that inequality

$$
\left[y_{2}(\omega)-y_{1}(\omega)\right]-\left[y_{2}(0)-y_{1}(0)\right]=\int_{0}^{\omega}\left[f\left(t, y_{2}(t)\right)-f\left(t, y_{1}(t)\right)\right] d t \geq 0 .
$$

To be periodic, the solution $y_{1}(t)$ from the domain above the curve $\gamma_{i}(t)$ must necessarily transfer into the domain located under the curve $\gamma_{i}(t)$. This means that there exists a time point $t^{\prime} \in[0, \omega]$ such that

$$
y_{1}\left(t^{\prime}\right)<\gamma_{i}\left(t^{\prime}\right)<y_{2}\left(t^{\prime}\right)
$$

But by theorem condition

$$
f\left(t^{\prime}, \gamma_{i}\left(t^{\prime}\right)\right)=0,
$$

and a function $\gamma_{i}(t)$ graph is the boundary of the domains of sign change for the function $f(t, x)$. Therefore, in time point $t^{\prime}$ the following inequality must be realized:

$$
f\left(t^{\prime}, y_{1}\left(t^{\prime}\right)\right)<f\left(t^{\prime}, y_{2}\left(t^{\prime}\right)\right) \text {. }
$$

From this inequality and continuity of the function $f(t, x)$ it follows that in (8) there is a sign of strict inequality, and this contradicts the assumption that the solutions $y_{1}(t)$ and $y_{2}(t)$ are $\omega$-periodic.

Thus, the obtained contradiction shows that in the interval

$$
\alpha_{i} \leq x \leq \beta_{i}
$$


there exists no more than one $\omega$-periodic solution of Equation (1). But, as follows from the Theorem 5, in this interval there necessarily exists at least one $\omega$-periodic solution of the Equation (1). Hence it can be finally concluded that in each interval

$$
\alpha_{i} \leq x \leq \beta_{i}, \quad i=1,2, \ldots, n,
$$

there is only one $\omega$-periodic solution of Equation (1). Since there are $n$ such intervals, then the Equation (1) has exactly $n \omega$-periodic solutions.

In particular, it follows from Theorem 5 that if the right-hand side of the Equation (1) is representable in the form

$$
f(t, x)=\prod_{i=1}^{n} f_{i}(t, x),
$$

where $f_{i}(t, x) \equiv 0$ for $x=\gamma_{i}(t), \frac{f_{i}(t, x)}{x-\gamma_{i}(t)}$ is of constant sign for $x \neq \gamma_{i}(t), i=1,2, \ldots, n$, then the Equation (1) has no less than $n \omega$-periodic solutions, if $\gamma_{i}(t), i=1,2, \ldots, n$ satisfy the requirements of Theorem 5 .

\section{Conclusions}

Using the apparatus of derived numbers allows to weaken the constraints imposed on the right-hand sides of the differential equations analyzed in this paper, and thereby increase the generality degree of the results. The upper and lower bounds for the numbers of almost periodic solutions of ordinary first-order differential equations are carried out. Conditions for the existence and stability of almost periodic solutions are established. Using the apparatus of derived numbers allowed us to expand the scope of the results obtained. Theorems of the derived numbers method to estimate the number of periodic solutions of first-order ordinary differential equations are formulated and proved.

Author Contributions: All authors are contributing equally to this research.

Funding: This research received no external funding.

Conflicts of Interest: The authors declare no conflict of interest.

\section{References}

1. Poincare, H. New Methods of Celestial Mechanics; Springer: Berlin, Germany, 1992; p. 772, ISBN 978-1-56396-117-5.

2. Lyapunov, A.M. The general problem of the stability of motion (translated into English by A. T. Fuller). Int. J. Control 1992, 55, 531-773, Also published as a book by Taylor \& Francis, London. [CrossRef]

3. Pliss, V.A. Nelockalnye Problemy Teorii Kolebaniy; Nauka: Moscow, Russian, 1964; p. 367. (In Russian)

4. Alferov, G.V.; Ivanov, G.G.; Efimova, P.A.; Sharlay, A.S. Stability of linear systems with Multitask Right-hand Member. In Stochastic Methods for Estimation and Problem Solving in Engineering; Kadry, S., Ed.; IGI Publisher: Hershey, PA, USA, 2018; pp. 74-112. [CrossRef]

5. Kadry, S.; Alferov, G.; Ivanov, G.; Sharlay, A. Almost Periodic Solutions of First-Order Ordinary Differential Equations. Mathematics 2018, 6, 171. [CrossRef]

6. Ivanov, G.; Alferov, G.; Efimova, P. Integrability of nonsmooth one-variable functions. In Proceedings of the 2017 Constructive Nonsmooth Analysis and Related Topics (Dedicated to the Memory of V.F. Demyanov) (CNSA), St. Petersburg, Russia, 22-27 May 2017.

7. Kadry, S.; Alferov, G.; Ivanov, G.; Sharlay, A. About stability of selector linear differential inclusions. AIP Conf. Proc. 2018, 2040, 150013.

8. Alferov, G.; Ivanov, G.; Efimova, P.; Sharlay, A. Study on the structure of limit invariant sets of stationary control systems with nonlinearity of hysteresis type. AIP Conf. Proc. 2017, 1863, 80003.

9. Ivanov, G.G.; Sharlay, A.S. On stability of linear homogeneous switched systems. In Proceedings of the 2015 International Conference "Stability and Control Processes" in Memory of V.I. Zubov (SCP), St. Petersburg, Russia, 5-9 October 2015; pp. 13-15. 
10. Malafeyev, O.; Nemnyugin, S.; Alferov, G. Charged particles beam focusing with uncontrollable changing parameters. In Proceedings of the 2014 2nd International Conference on Emission Electronics (ICEE), St. Petersburg, Russia, 30 June-4 July 2014; pp. 1-3.

11. Kadry, S.; Alferov, G.; Ivanov, G.; Sharlay, A. Stabilization of the program motion of control object with elastically connected elements. AIP Conf. Proc. 2018, 2040, 150014.

12. Pichugin, Y.; Alferov, G.; Malafeyev, O. Parameters estimation in mechanism design. Contemp. Eng. Sci. 2016, 9, 175-185. [CrossRef]

13. Ivanov, G.G.; Alferov, G.V.; Sharlay, A.S.; Efimova, P.A. Conditions of Asymptotic Stability for Linear Homogeneous Switched Systems. AIP Conf. Proc. 2017, 1863, 080002. [CrossRef]

14. Alferov, G.V.; Ivanov, G.G.; Efimova, P.A. The Structural Study of Limited Invariant Sets of Relay Stabilized Systems. In Mechanical Systems: Research, Applications and Technology; Nova Science Publishers, Inc.: Hauppauge, NY, USA, 2017; pp. 101-164.

15. Malafeyev, O.; Neverova, E.; Nemnyugin, S.; Alferov, G. Multi-criteria model of laser radiation control. In Proceedings of the 2014 2nd International Conference on Emission Electronics (ICEE), St. Petersburg, Russia, 30 June-4 July 2014; pp. 1-5.

16. Kulakov, F.; Alferov, G.V.; Efimova, P.; Chernakova, S.; Shymanchuk, D. Modeling and Control of Robot Manipulators with the Constraints at the Moving Objects. In Proceedings of the 2015 International Conference "Stability and Control Processes" in Memory of V.I. Zubov (SCP), St. Petersburg, Russia, 5-9 October 2015; pp. 102-105.

17. Kulakov, F.; Alferov, G.; Efimova, P. Methods of remote control over space robots. In Proceedings of the 2015 International Conference on Mechanics—Seventh Polyakhov's Reading, St. Petersburg, Russia, 2-6 February 2015.

18. Efimova, P.; Shymanchuk, D. Dynamic Model of Space Robot Manipulator. Appl. Math. Sci. 2015, 94, 4653-4659. [CrossRef]

19. Kulakov, F.; Sokolov, B.; Shalyto, A.; Alferov, G. Robot Master Slave and Supervisory Control with Large Time Delays of Control Signals and Feedback. Appl. Math. Sci. 2016, 10, 1783-1796. [CrossRef]

20. Alferov, G.V.; Malafeyev, O.A.; Alferov, G. The robot control strategy in a domain with dynamical obstacles. In Reasoning with Uncertainty in Robotics; RUR 1995, Lecture Notes in Computer Science (Lecture Notes in Artificial Intelligence); Springer: Berlin/Heidelberg, Germany, 1996; Volume 1093, pp. 209-217.

21. Kulakov, F.; Kadry, S.; Alferov, G.; Sharlay, A. Bilateral remote control over space manipulators. AIP Conf. Proc. 2018, 2040, 150015.

22. Kulakov, F.; Alferov, G.; Sokolov, B.; Gorovenko, P.; Sharlay, A. Dynamic analysis of space robot remote control system. AIP Conf. Proc. 2018, 1959, 080014.

23. Kadry, S.; Alferov, G.; Kondratyuk, A.; Kurochkin, V.; Zhao, S. Modeling the motion of a space manipulation robot using position control. AIP Conf. Proc. 2019.

24. Kulakov, F.; Kadry, S.; Alferov, G.; Efimova, P. Remote Control of Space Robots Change-Adaptive in Its External Environment. Int. J. Online Biomed. Eng. 2019, 15, 84-98. [CrossRef]

25. Alferov, G.; Gorizontov, A. Resource management in flexible automated production. Kibern. Vychislitel'naya Tek. 1987, 73, 76-81.

26. Alferov, G.; Malafeyev, O.; Maltseva, A. Game-theoretic model of inspection by anti-corruption group. AIP Conf. Proc. 2015, 1648, 450009.

27. Neverova, E.G.; Malafeyev, O.A.; Alferov, G.V.; Smirnova, T.E. Model of interaction between anticorruption authorities and corruption groups. In Proceedings of the Model of Interaction between Anticorruption Authorities and Corruption Groups, St. Petersburg, Russia, 5-9 October 2015; pp. 488-490.

28. Alferov, G.V.; Malafeyev, O.A.; Maltseva, A.S. Programming the Robot in Tasks of Inspection and Interception. In Proceedings of the 2015 International Conference on Mechanics-Seventh Polyakhov's Reading, St. Petersburg, Russia, 2-6 February 2015.

29. Malafeyev, O.; Alferov, G.; Andreyeva, M. Group strategy of robots in game-theoretic model of interception with incomplete information. In Proceedings of the 2015 International Conference on Mechanics-Seventh Polyakhov's Reading, St. Petersburg, Russia, 2-6 February 2015; pp. 1-3.

30. Knobloch, H.W. An existence theorem for periodic solutions of nonlinear ordinary differential equations. Mich. Math. J. 1962, 9, 303-309. [CrossRef]

31. Mawhin, J. Periodic solutions of nonlinear functional differential equations. J. Differ. Equ. 1971, 10, $240-261$. [CrossRef] 
32. Kadry, S.; Alferov, G.; Ivanov, G.; Sharlay, A. Derived numbers of one variable monotonic functions. Ital. J. Pure Appl. Math. 2019, 41, 637-648.

33. Kadry, S.; Alferov, G.; Ivanov, G.; Sharlay, A. Derived numbers of one variable convex functions. Ital. J. Pure Appl. Math. 2019, 41, 649-662.

34. Pliss, V.A. On the number of periodic solutions of an equation with a polynomial right-hand side. DAN USSR $1959,127,965-968$. 